\title{
EFEITO DA INUNDAÇÃO LATERAL SOBRE A DISTRIBUIÇÃO DA VEGETAÇÃO RIPÁRIA EM UM TRECHO DO RIO CUIABÁ, MT ${ }^{1}$
}

\author{
Ricardo Keichi Umetsu², Pierre Girard³, Dalva Maria da Silva Matos e Carolina Joana da Silva² \\ RESUMO - Estudos hidrológicos e fitossociológicos foram realizados num perfil topográfico de 550 m instalado \\ perpendicularmente ao rio Cuiabá, no Município de Rosário Oeste, MT, para analisar a influência da inundação \\ sobre a distribuição da vegetação ripária. Utilizando os dados fluviométricos da estação de Rosário Oeste de \\ 1966 a 2003, um modelo hidrológico de remanso em regime subcrítico foi ajustado, permitindo estabelecer \\ a série hidrológica na área de estudo. A partir dessa série, os intervalos de recorrência nessa área foram obtidos. \\ Os resultados sugeriram que a margem do canal principal, o canal secundário e o dique marginal, a planície \\ de inundação e o terraço alagam a cada $\sim 1-1,7$ ano, $\sim 1,77-2,8$ anos, $\sim 3$ anos e de $\sim 3,25$ a $\sim 39$ anos, respectivamente. \\ A espécie Combretum leprosum Mart. (Combretaceae) apresentou maior VI na margem do canal principal, \\ Callisthene fasciculata (Spr.) Mart. (Vochysiaceae) na margem do canal principal e no terraço e Licania parvifolia \\ Huber (Chrysobalanaceae), Cariniana estrellensis (Raddi) Kuntze. (Lecythidaceae) e Vochysia divergens Pohl. \\ (Vochysiaceae) na planície de inundação. Os resultados indicaram que a frequência e, principalmente, o tempo \\ de alagamento são os principais determinantes ecológicos da distribuição das espécies vegetais ao longo do \\ perfil topográfico.
}

Palavras-chave: Fatores limitantes, Fitossociologia e Hidroperíodo.

\section{EFFECT OF LATERAL FLOODING ON RIPARIAN VEGETATION DISTRIBUTION IN A RIVER CUIABÁ STRECH, MT, BRAZIL}

\begin{abstract}
Hydrological and phytosociological studies were conducted in a $550 \mathrm{~m}$ topographic profile installed perpendicularly to the Cuiabá River, in the municipality of Rosário Oeste, MT, Brazil, in order to analyze the influence of flooding on riparian vegetation distribution. A backwater hydrological model under a subcritical regime was fitted allowing the establishment of a hydrological series in the study area. From this series, the recurrence intervals for the area were obtained. The results suggested that the banks of the main channel, the secondary channel and its lateral dike, the flood plain, and the terrace are flooded at every $\sim 1-1.7$ years, $\sim 1.77-2.8$ years, $\sim 3$ years, and from $\sim 3.25$ to $\sim 39$ years, respectively. The species Combretum leprosum Mart. (Combretaceae) presented the greatest VI in the bank of the main channel; Callisthene fasciculata (Spr.) Mart. (Vochysiaceae) presented the greatest VI in the bank of the main channel and in the terrace; and Licania parvifolia Huber (Chrysobalanaceae), Cariniana estrellensis (Raddi) Kuntze. (Lecythidaceae), and Vochysia divergens Pohl. (Vochysiaceae) in the flood plain. The results indicated that flooding frequency and especially the flooding time are the main determinative ecological factors of plant species distribution along the topographic profile.
\end{abstract}

Keywords: Hydroperiod, Limiting factors and Phytosociology.

\footnotetext{
${ }^{1}$ Recebido em 28.04.2009 e aceito para publicação em 02.05.2011.

${ }^{2}$ Universidade do Estado de Mato Grosso, UNEMAT, Brasil. E-mail: <rkumetsu@yahoo.com.br> e <ecopanta@terra.com.br>.

${ }^{3}$ Centro de Pesquisa do Pantanal, CPP, Brasil. E-mail: <pierreg@cpd.ufmt.br>.

${ }^{4}$ Departamento de Botânica, Universidade Federal de São Carlos, UFSCAR, Brasil. E-mail: <dmatos@power.ufscar.br> .
} 


\section{INTRODUÇÃO}

Inundações são eventos de extrema importância para a manutenção de ecossistemas ripários (GREGORY et al., 1991; POFF et al., 1997; NILSSON; SVEDMARK, 2002). O hidroperíodo (magnitude, duração e frequência de inundação) é considerado importante fator físico de controle da distribuição de espécies vegetais ripárias e de outras áreas úmidas (MITSCH; GOSSELINK, 1993; HUPP; OSTERKAMP, 1996). O ambiente físico-químico resultante dos processos de inundação causa respostas morfológicas, anatômicas, fisiológicas, fenológicas e, ou, adaptações etológicas na biota, além de produzir estruturas de comunidade características (JUNK, 1999).

O regime fluvial condiciona as planícies de inundação e determina a evolução sucessional das comunidades de plantas ripárias e processos ecológicos (NILSSON; SVEDMARK, 2002). Quando naturalmente variável, cria e mantém a dinâmica dentro do canal e da planície de inundação e condiciona hábitats que são essenciais às espécies aquáticas e ripárias (POFF et al., 1997). A saúde das comunidades ripárias é dependente dessas características hidrológicas, e a importância das inundações periódicas é amplamente reconhecida.

A maioria, entretanto, dos grandes rios do mundo possui regime hídrico regulado por barragens (DYNESIUS; NILSSON, 1994. Estas, utilizadas para a geração de energia hidroelétrica e controle de inundações, alteram o hidroperíodo fluvial, afetando principalmente as grandes inundações, o que pode causar profundos impactos nas comunidades vegetais ripárias (POLZIN; ROOD, 2000). Portanto, devido à grande importância dos ecossistemas ripários, torna-se necessário o conhecimento do funcionamento desses sistemas através da avaliação da influência do hidroperíodo sobre as comunidades vegetais ripárias. Trabalhos realizados por Teversham e Slaymaker (1976), Bedinger (1979), Bren e Gibbs (1986), Hughes (1990), Hupp e Osterkamp (1996), Ferreira (1997), Ferreira (2000) e Chapin et al. (2002) demonstram que a estrutura da comunidade vegetal ripária é influenciada pela inundação.

O rio Cuiabá possui grandes importâncias econômica e ambiental, é formado pela confluência dos rios Manso e Cuiabazinho e é importante tributário da bacia do Alto Paraguai e, portanto, do Pantanal Mato-Grossense. Entretanto, em 1999, no rio Manso, começou a operar a Hidroelétrica APM (Aproveitamento Múltiplo) Manso e, desde então, passou a ter sua vazão controlada. Portanto, espera-se aumentar o conhecimento sobre esses sistemas, o que permitirá promover medidas de conservação e recuperação desses ambientes e criar subsídios para o estudo de longo prazo sobre o efeito de barragens sobre a vegetação ripária.

Dessa forma, este estudo teve como objetivo verificar a influência da magnitude, frequência e duração da inundação lateral sobre a distribuição da vegetação ripária em um trecho do rio Cuiabá, Mato Grosso.

\section{MATERIAL E MÉTODOS}

\section{1. Área de estudo}

O estudo foi realizado em um trecho da margem direita do rio Cuiabá, nas coordenadas geográficas 1447’23.200" S e 56¹9’10.008" O, próximo à cidade de Rosário Oeste, Mato Grosso (Figura 1). De acordo com PCBAP (1997), a bacia do rio Cuiabá ocupa uma área de 28.732 km², com 841 km de perímetro pertencente à bacia do Alto Rio Paraguai, e, segundo Alvarenga et al. (1984), faz parte do grande sistema hidrológico Paraguai-Paraná-Prata.

O clima da região, pela classificação climática de Köppen, é do tipo Aw (tropical semiúmido), com sazonalidade marcada por dois períodos bem distintos, inverno seco e verão chuvoso. Os meses de maio a setembro são secos e os meses de outubro a abril, chuvosos (GIRARD, 2003). A formação geológica da bacia e o clima regional predominante conduzem a padrões sazonais com elevada precipitação e que contribuem para inundações no Alto Pantanal (SEVERI, 1999).

Segundo Brasil (1982), no médio curso do rio Cuiabá predomina vegetação do tipo campo-cerrado cerradão. A área de estudo no Município de Rosário Oeste, MT, tem como características a vegetação primária e encontra-se o mais próximo possível de uma estação fluviométrica, que dispunha de hidrogramas antigos. Os valores de cota da estação foram, então, transportados para a área de estudo, que está a montante da estação, para então determinar a extensão, magnitude, frequência e tempo de alagamento.

\subsection{Aquisição de dados fluviométricos}

Os dados fluviométricos da Estação Rosário Oeste (cód. 66250001) foram adquiridos através da Secretaria de Defesa Civil do Estado de Mato Grosso e da Agência

Revista Árvore, Viçosa-MG, v.35, n.5, p.1077-1087, 2011 


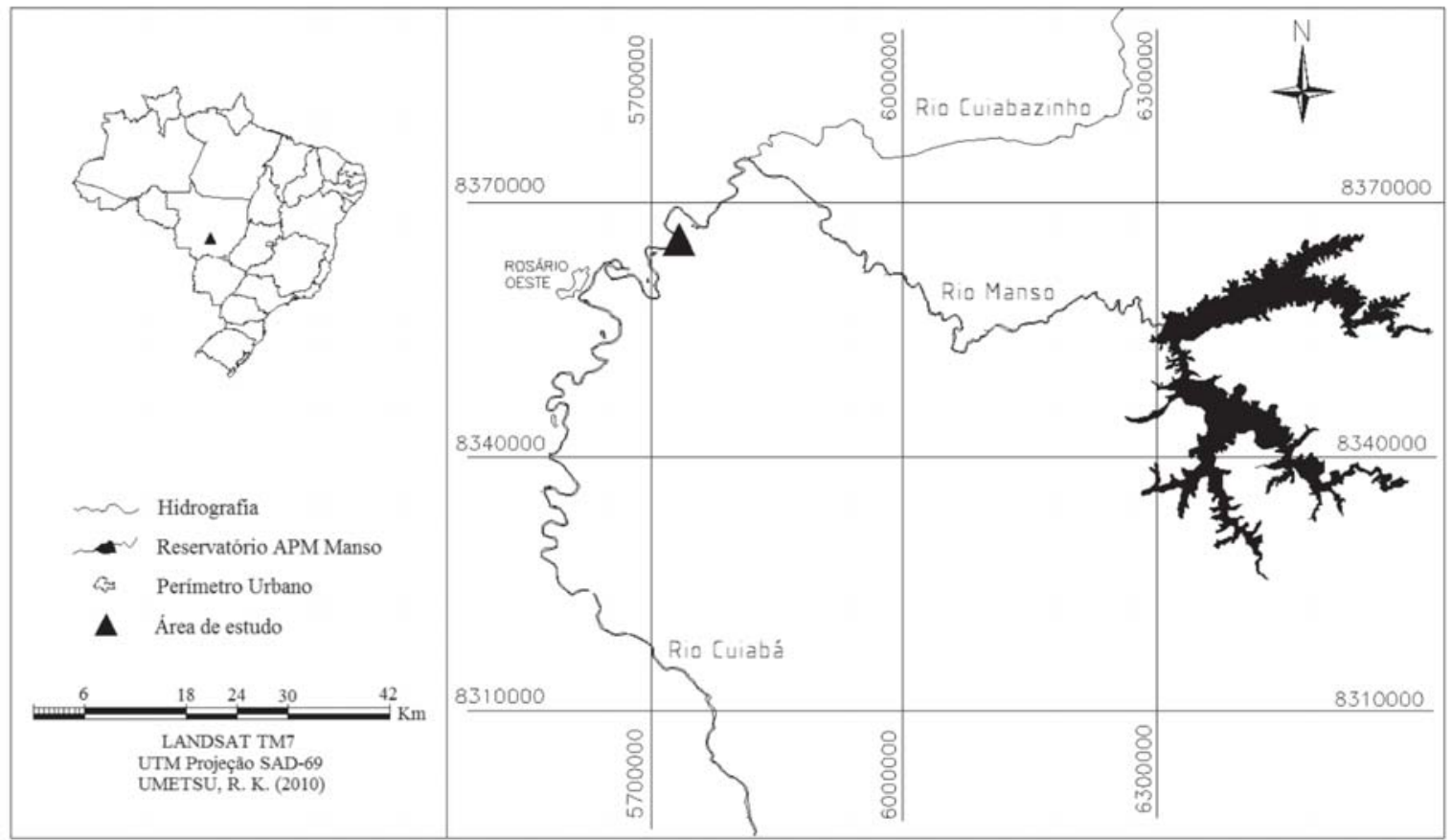

Figura 1 - Área de estudo, localidade de Rosário Oeste, MT. Figure 1 - Study area, locality of Rosário Oeste, MT, Brazil.

Nacional das Águas - ANA (http://hidroweb.ana.gov.br/ HidroWeb). A série comporta dados fluviométricos diários de cota e vazão entre 1966 e 2003, com algumas falhas. A série bruta foi utilizada para o cálculo dos intervalos de recorrência. Essa estação possui área de drenagem de $15.908 \mathrm{~km}^{2}$, sendo de responsabilidade da ANA e tendo a empresa Furnas como operadora.

\subsection{Frequência das cheias}

Para o estudo das frequências de cheias foram utilizados intervalos de recorrências calculados segundo Christofoletti (1981). Para isso, utilizou-se a seguinte equação: $\mathrm{Ir}=\mathrm{N}+1 / \mathrm{M}$, em que Ir é o intervalo de recorrência, $\mathrm{N}$ o número total dos eventos considerados na série anual e $\mathrm{M}$ o número de ordem que representa a posição da cheia na escala organizada.

\subsection{Levantamento do perfil topográfico em cotas altimétricas reais}

O perfil topográfico na área selecionada, feito perpendicularmente à margem do rio, foi determinado através do procedimento de topografia-padrão (teodolito) e régua topográfica, a partir da base geodésica instalada no local (RN - Registro de nível). Os primeiros pontos topográficos foram instalados conforme a geomorfologia fluvial para demonstrar o canal principal, canal secundário e dique marginal; os pontos restantes foram instalados de 50 em 50 m, totalizando um perfil topográfico de 550 m. A cota altimétrica real foi obtida através de GPS - Geodésico. Na estação fluviométrica foi determinada a altitude real, através de GPS - Geodésico (SAD-69). A altitude foi calculada com referência a um "RN" (Registro de nível) que determina a altura do ponto zero das réguas linimétricas. A partir disso foi possível determinar as cotas fluviométricas nos hidrogramas em cotas altimétricas reais.

\subsection{Descrição da seção transversal}

Para o cálculo da linha de água a montante da estação fluviométrica, na área de estudo, foi preciso determinar a seção transversal do rio no local onde estava a estação e também na área de estudo. Para tanto foi realizado um estudo batimétrico, em que, com o auxílio de um barco e guincho batimétrico, foi determinada a profundidade em intervalos de $5 \mathrm{~m}$, bem como a largura e vazão do rio naquele momento. 


\subsection{Determinação do nível de água na área de estudo}

As cotas conhecidas na estação fluviométrica foram utilizadas para determinar o nível de água na área de estudo. Para tanto, equações hidrológicas foram utilizadas e calculadas através do programa HEC-RAS 3.1.1 - U.S. Army Corps of Enginners. Segundo Tucci (2001), o cálculo da linha de água em regime subcrítico é realizado de jusante para montante. Essa é a condição da maioria dos rios naturais e calculada através da seguinte equação:

$$
\begin{aligned}
\mathrm{Z} 1= & \mathrm{Z} 2+\mathrm{Q}^{2} / 2\left[1 / \mathrm{g}\left(\alpha 2 / \mathrm{A}_{2}{ }^{2}-\alpha 1 / \mathrm{A}_{1}{ }^{2}\right)+\mathrm{C} \mid(\alpha 1 /\right. \\
& \left.\left.\mathrm{A}_{1}{ }^{2}-\alpha 2 / \mathrm{A}_{2}{ }^{2}\right) \mid+\Delta \mathrm{x}\left(1 / \mathrm{Kc}^{2}+1 / \mathrm{Kc}^{2}\right)\right]
\end{aligned}
$$

em que Z1 e Z2 são as cotas da seção, $Q$ a vazão, $\alpha_{i}$ o coeficiente de Coriolis das seções, A a área do perfil transversal, C o coeficiente de contração ou expansão, Äx a distância entre as seções e $K c=\left(A \cdot R^{2 / 3}\right) / n(e m$ que "R" é o raio hidráulico e "n”, o coeficiente de rugosidade de Manning). Nessa equação, as incógnitas são Z1, A1 e R1. Pode-se estabelecer a relação entre $\mathrm{Z1}$ = $\mathrm{f}(\mathrm{A} 1)$ e $\mathrm{Z1}$ = $\mathrm{f}(\mathrm{R} 1)$ com base na seção transversal, e a única incógnita passa a ser $\mathrm{Z} 1$, que é a cota da seção a montante.

O cálculo hidrológico foi calibrado através de observações diretas entre a cota na estação fluviométrica e a cota na área de estudo. Conhecidas as cotas do rio Cuiabá na área de estudo, puderam-se determinar a extensão e frequência de alagamento, que foram avaliadas da seguinte maneira: a cota mínima para a água transbordar do canal (extensão de alagamento $=0$ ) foi determinada a partir da comparação entre o perfil topográfico e as cotas fluviométricas e da mesma maneira para a cota fluviométrica máxima que corresponder à extensão do alagamento.

\subsection{Fitossociologia}

Para o levantamento fitossociológico foram demarcadas sobre o transecto de $550 \mathrm{~m} 10$ parcelas de 50 x 20 m subdivididas em 100 parcelas de 5 x 20 m, de forma contígua e não aleatória, totalizando 1 ha $\left(10.000 \mathrm{~m}^{2}\right)$. Transectos são métodos de amostragem em que se imagina que comunidades são fortemente influenciadas por um gradiente ambiental (BARBOUR et al., 1987). Foram amostrados todos os indivíduos arbustivo-arbóreos com circunferência à altura do peito superior ou igual a $5 \mathrm{~cm}(\mathrm{CAP} \geq 5 \mathrm{~cm})$. Os indivíduos foram identificados através da comparação de material botânico herborizado na coleção do herbário da Universidade Federal de Mato
Grosso. Os nomes científicos e os respectivos autores foram confirmados e atualizados através do uso de bibliografia e consultas ao site do Museu Botânico de Missouri (http://www.tropicos.org). O sistema de classificação utilizado foi o APG II (Angiosperm Phylogeny Group II) (APG II, 2003). Os parâmetros fitossociológicos, para a obtenção do VI - Valor de Importância, foram calculados segundo a metodologia descrita por MuellerDombois e Elemberg (1974). Também, foram calculados o índice de diversidade de Shannon (H') e a equabilidade de Pielou (J') (BROWER; ZAR, 1984). Para a análise de similaridade entre as parcelas do transecto foi utilizada a análise de Cluster (GAUCH, 1982) com Distância Relativa de Sorensen ligadas pelo método de simples ligação ou do vizinho mais próximo.

\section{RESULTADOS}

\subsection{Levantamento geodésico}

Os resultados dos levantamentos geodésicos estão sintetizados na Figura 2. O gradiente de inundação encontra-se na margem direita do rio e apresenta leve depressão onde a água fica retida após inundações.

\subsection{Frequência das cheias na estação fluviométrica de Rosário Oeste-MT}

A análise da frequência das cheias possibilitou determinar o intervalo de suas recorrências nos anos de 1966 a 2003, permitindo extrapolar a magnitude das

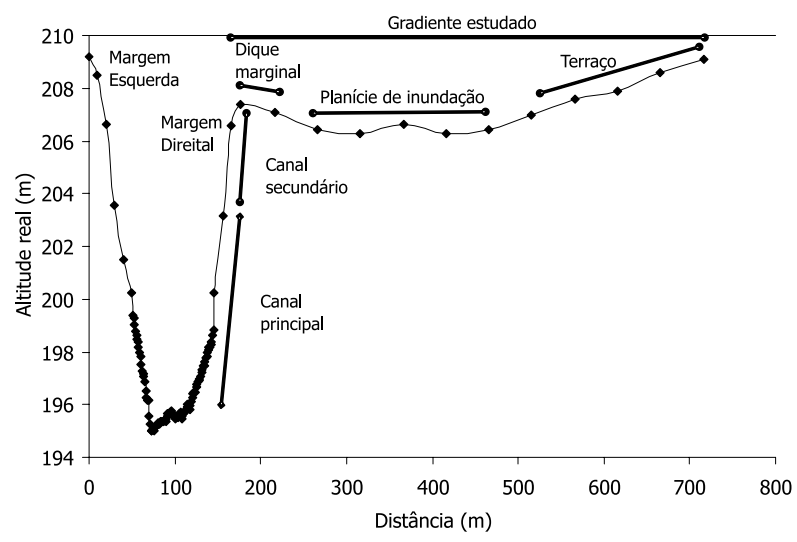

Figura 2 - Perfil topográfico em altitude real e descrição da geomorfologia fluvial na área de estudo, Rosário Oeste, MT.

Figure 2 - Topographic profile in real altitude and river geomorphology description in the study area, Rosário Oeste, MT, Brazil. 
cheias para os mais variados intervalos. A maior cheia registrada $\left(2.285 \mathrm{~m}^{3} . \mathrm{s}^{-1}\right)$ apresentou intervalo de recorrência de 39 anos, seguido pela cheia de $2.188 \mathrm{~m}^{3} . \mathrm{s}^{-1}$, com intervalo de 19,5 anos. A menor cheia registrada $\left(954 \mathrm{~m}^{3} . \mathrm{s}^{-1}\right)$ obteve intervalo de 1,03 ano.

\subsection{Cálculo do nível d’água na área de estudo}

O levantamento geodésico permitiu estabelecer a declividade entre as seções da área de estudo e da estação fluviométrica de Rosário Oeste, MT, obtendo-se o valor de 0,222331 m. $\mathrm{km}^{-1}$, sendo a distância entre a seções de $26,51 \mathrm{~km}$, obtida através de ferramentas de SIG em mapa digitalizado. O cálculo no nível d’água na área de estudo foi calibrado através de observações diretas na cota da estação fluviométrica e na área de estudo. A obtenção desses valores permitiu o cálculo da linha de água em regime subcrítico, obtendo-se, assim, o valor da cota na estação fluviométrica de Rosário Oeste que reflete no transbordamento do canal na área de estudo e, dessa forma, o enchimento da planície de inundação. A cota obtida foi de $7 \mathrm{~m}$, ou seja, 202,645 m, representando uma vazão de $1763 \mathrm{~m}^{3} . \mathrm{s}^{-1}$ e intervalo de recorrência de três anos. Essa cota reflete na área de estudo a altitude de 207,4 m do nível da água e extensão de alagamento, a partir do dique marginal de $\sim 375,46 \mathrm{~m}$.

\subsection{Mapeamento da freqüência de inundação}

O cálculo do nível d’água na área de estudo através do modelo de remanso e o cálculo do intervalo de recorrência da inundação permitiram mapear a frequência de inundação no perfil topográfico (Figura 3). A frequência de inundação que corresponde a 3 anos e compreende a inundação da planície atinge a extensão de alagamento de $\sim 375,46 \mathrm{~m}$ a partir do dique marginal. A frequência de $~ 39$ anos corresponde a uma extensão de $\sim 495,25 \mathrm{~m}$. As parcelas 3, 6 e partes das parcelas 4 e 5 sofrem maior influência da alta frequência e duração da inundação, quando comparadas com outras parcelas.

\subsection{Fitossociologia}

Foram amostrados 1.072 indivíduos, distribuídos em 68 espécies e 30 famílias. A parcela 2 apresentou maior número de indivíduos (189 ind.), seguida pelas parcelas 9 (181 ind.), 4 (179 ind.), 5 (139 ind.), 3 (116 ind.), 10 (110 ind.), 6 (91 ind.) e 1 (67 ind.), e as parcelas 7 e 8 não apresentaram indivíduos com CAP $\geq 5 \mathrm{~cm}$. As parcelas 2 e 9 exibiram 25 espécies, seguidas pelas parcelas 1, 5, 4, 3, 10 e 4 com 23, 21, 19, 11, 9 e 4 espécies,

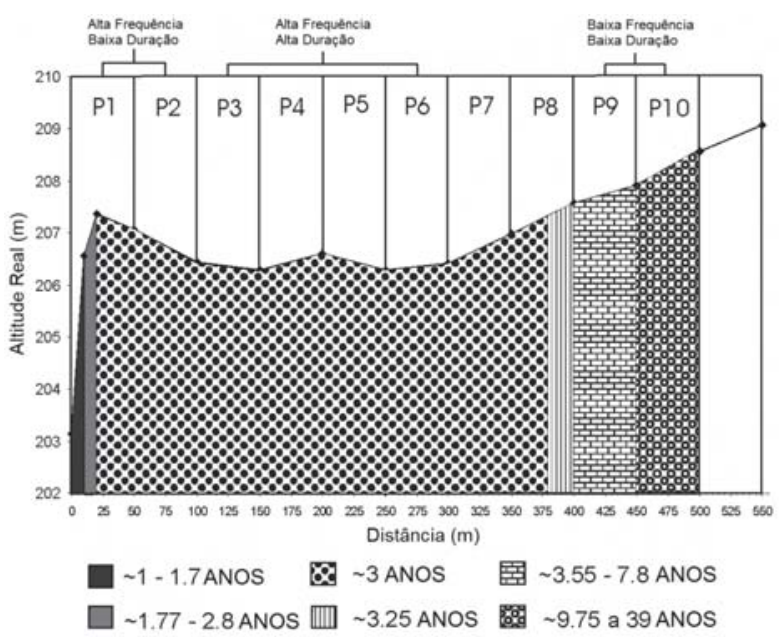

Figura 3 - Mapeamento da frequência de inundação na área de estudo.

Figure 3 - Flooding frequency mapping for the study area, Rosário Oeste, MT, Brazil.

respectivamente. O maior valor de diversidade ocorreu na parcela 2, com 2,60 nats.ind ${ }^{-1}$ e equabilidade 0,81 , seguido pelas parcelas $1\left(\mathrm{H}^{\prime}=2,53\right.$ nats.ind $\left.^{-1} \mathrm{e} \mathrm{J}^{\prime}=0,81\right)$, $5\left(\mathrm{H}^{\prime}=2,40\right.$ nats.ind $\left.{ }^{-1} \mathrm{e} \mathrm{J}^{\prime}=0,79\right), 9\left(\mathrm{H}^{\prime}=2,32\right.$ nats.ind $^{-1} \mathrm{e}$ $\left.\mathrm{J}^{\prime}=0,72\right), 4\left(\mathrm{H}^{\prime}=2,29\right.$ nats.ind $\left.^{-1} \mathrm{e} \mathrm{J}^{\prime}=0,78\right), 3\left(\mathrm{H}^{\prime}=1,96\right.$ nats.ind ${ }^{-1}$ e J' $\left.=0,82\right), 10\left(H^{\prime}=1,32\right.$ nats.ind ${ }^{-1}$ e J' $\left.=0,60\right)$ e $6\left(H^{\prime}=0,43\right.$ nats.ind ${ }^{-1}$ e J' $\left.=0,31\right)$.

Através do cálculo dos parâmetros fitossociológicos, foi possível avaliar a distribuição das espécies arbustivo-arbóreas das parcelas inseridas no gradiente estudado (Figura 4). Na parcela 1, a espécie Combretum leprosum Mart. apresentou maior valor de importância. A espécie Callisthene fasciculata Mart. apresentou maior VI na parcela 2. Na parcela 3, a espécie Licania parvifolia Huber. seguida pelos indivíduos encontrados mortos apresentou maior VI. A espécie Cariniana estrellensis (Raddi) Kuntze, também seguida pelos indivíduos mortos, apresentou, na parcela 4, o maior valor de importância. Nas parcelas 5 e 6, a espécie Vochysia divergens Pohl. apresentou maior valor de importância. Da mesma forma que na parcela 2, a espécie C. fasciculata mostrou maior VI na parcela 9. Na parcela 10, a espécie Machaerium hirtum (Vell.) Stellfeld seguida pela espécie C. fasciculata teve maior VI.

$\mathrm{Na}$ análise de similaridade florística entre as parcelas (Figura 5) foi possível verificar a formação de dois grupos. Um formado pelas parcelas 1 , 2 e 
9 e outro pelas parcelas 3, 4, 5 e 6, e a parcela 10 apresentou menor similaridade entre os dois grupos formados.

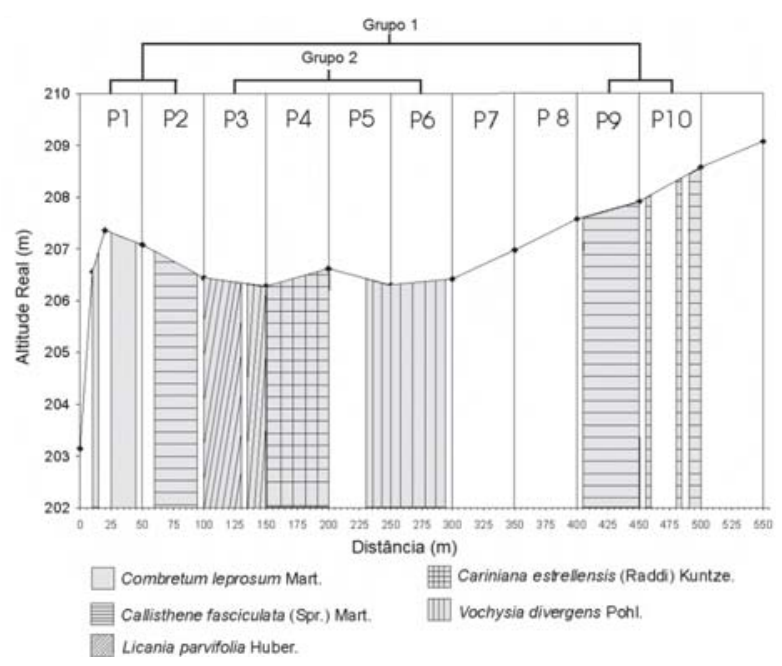

Figura 4 - Distribuição das espécies arbustivo-arbóreas com maior IVI, no perfil topográfico, na área de estudo, Rosário Oeste, MT.

Figure 4-Distribution of shrub and tree species with highest $I V$ in the topographic profile, in the study area, Rosário Oeste, MT, Brazil.

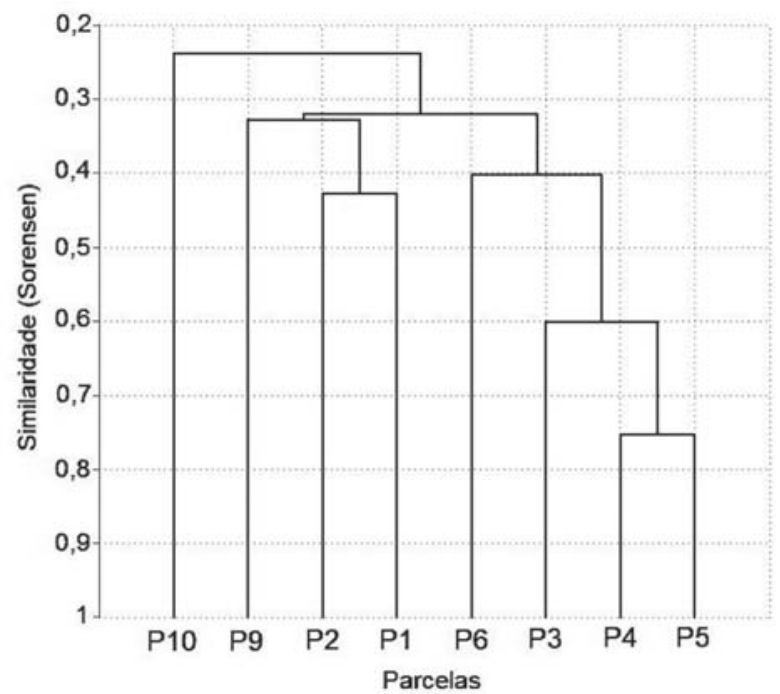

Figura 5 - Análise de similaridade da vegetação ripária da área de estudo, Rosário Oeste, MT.

Figure 5 - Similarity analysis for riparian vegetation in the study area, Rosário Oeste, MT, Brazil.

\section{DISCUSSÃO}

Nas últimas décadas, muitos estudos realizados sobre a influência da inundação em sistemas ripários têm demonstrado a importância desse evento natural sobre a estrutura e função das florestas ripárias. O transbordamento do dique marginal e o alagamento das margens dos rios provocam condições adversas à sobrevivência das espécies vegetais, o que condiciona características específicas das florestas ripárias.

Através da abordagem utilizada neste estudo foi possível verificar a relação entre a inundação lateral e a distribuição da vegetação no gradiente de inundação. A utilização de hidrogramas antigos permitiu avaliar o comportamento hidrológico na área de estudo, e com isso foi possível também avaliar a magnitude, frequência e duração da inundação no gradiente lateral. A presença das espécies arbustivo-arbóreas encontradas em cada parcela reflete as condições ecológicas em que elas estão suscetíveis.

As parcelas 1, 2 e 9 apresentaram frequências de inundação relativamente altas, mas com baixo tempo de duração. mostraram maior diversidade e abundância de indivíduos que as parcelas 3, 4, 5, e 6, que apresentaram alta duração de alagamento. Essa tendência encontrada em áreas com alto tempo de indundação foi detectada por Lieberman et al. (1985), Ferreira $(1997,2000)$ e Ferreira e Almeida (2005). A inundação configura-se como barreira para a colonização da área por espécies de formações vegetais vizinhas, resultando em formação com baixa riqueza e diversidade.

Campbell et al. (1992) estudaram três áreas de diques geomorfológicos sequenciais das várzeas do rio Juruá, com idades conhecidas, e constataram que a área mais próxima ao leito do rio (mais inundável) possuía menor quantidade de espécies e baixa diversidade. As outras duas áreas tinham diversidade diretamente proporcional à idade e inversamente proporcional ao nível de inundação a que estavam sujeitas.

A composição florística e estrutural foi bastante distinta entre as parcelas com alto e baixo tempo de alagamento. A formação de dois grupos na análise de agrupamento, um com as parcelas com baixo tempo de alagamento (P1, P2, P9 e P10) e outro com parcelas com alto tempo de alagamento (P3, P4, P5 e P6), evidenciou esse fato. As parcelas submetidas ao maior tempo de inundação são colonizadas por espécies adaptadas a essa condição hidrológica, como Licania parvifolia,

Revista Árvore, Viçosa-MG, v.35, n.5, p.1077-1087, 2011 
Tabela 1 - Distribuição das espécies arbustivo-arbóreas amostradas nas parcelas estudadas em um trecho de floresta ripária do rio Cuiabá, Rosário Oeste, MT.

Table 1 - Shrub and tree species distribution recorded in studied plots in a riparian forest stretch along the Cuiabá River, Rosário Oeste, MT, Brazil.

\begin{tabular}{|c|c|c|c|c|c|c|c|c|c|}
\hline \multirow[t]{2}{*}{ Família } & \multirow[t]{2}{*}{ Espécie } & \multicolumn{8}{|c|}{ Parcelas } \\
\hline & & $\mathrm{P} 1$ & $\mathrm{P} 2$ & P3 & $\mathrm{P} 4$ & $\mathrm{P} 5$ & P6 & P9 & $\mathrm{P} 10$ \\
\hline \multirow{3}{*}{ Anacardiaceae } & Astronium fraxinifolium Schott ex Spreng. & $\mathrm{x}$ & & & & $\mathrm{x}$ & & & \\
\hline & Myracrodruon urundeuva Allemão & $\mathrm{x}$ & & & & & & $\mathrm{x}$ & \\
\hline & Spondia lutea L. & $\mathrm{x}$ & & & & & & & \\
\hline \multirow[t]{2}{*}{ Apocynaceae } & Aspidosperma australe Müll. Arg. & & & & & & & $\mathrm{x}$ & \\
\hline & Aspidosperma cylindrocarpon Müll. Arg. & & & & & & & $\mathrm{x}$ & \\
\hline \multirow[t]{6}{*}{ Bignoniaceae } & Jacaranda cuspidifolia Mart. & & & & $\mathrm{x}$ & & & $\mathrm{x}$ & \\
\hline & Jacaranda mimosifolia D. Don & & $\mathrm{x}$ & & & & & & $\mathrm{x}$ \\
\hline & $\begin{array}{l}\text { Tabebuia aurea (Silva Manso) Benth. \& Hook. } \\
\text { f. ex S. Moore }\end{array}$ & & $\mathrm{x}$ & & $\mathrm{x}$ & $\mathrm{x}$ & & $\mathrm{x}$ & $\mathrm{x}$ \\
\hline & Tabebuia avellanedae Lorentz ex Griseb. & & & & & & & $\mathrm{x}$ & \\
\hline & Tabebuia impetiginosa (Mart. ex DC.) Standl. & & $\mathrm{x}$ & & & & & & \\
\hline & Tabebuia roseoalba (Ridl.) Sandwith & $\mathrm{x}$ & $\mathrm{x}$ & & & & & $\mathrm{x}$ & \\
\hline Boraginaceae & Cordia maidophila Johnston & $\mathrm{x}$ & $\mathrm{x}$ & & & & & & \\
\hline Burseraceae & Protium heptaphyllum (Aubl.) Marchand & & & $\mathrm{x}$ & $\mathrm{x}$ & $\mathrm{x}$ & & & \\
\hline Celastraceae & Maytenus sp. & & & & $\mathrm{x}$ & & & & \\
\hline Chrysobalanaceae & Licania parvifolia Huber. & & $\mathrm{x}$ & & $\mathrm{x}$ & $\mathrm{x}$ & $\mathrm{x}$ & & \\
\hline \multirow[t]{2}{*}{ Clusiaceae } & Calophyllum brasiliense Cambess. & & & $\mathrm{x}$ & $\mathrm{x}$ & $\mathrm{x}$ & & & \\
\hline & Rheedia brasiliensis (Mart.) Planch. \& Triana & & & $\mathrm{x}$ & & & & & \\
\hline Combretaceae & Combretum leprosum Mart. & $\mathrm{x}$ & $\mathrm{x}$ & & & & & & \\
\hline Dilleniaceae & Curatella americana $\mathrm{L}$. & $\mathrm{x}$ & $\mathrm{x}$ & & $\mathrm{x}$ & & & $\mathrm{x}$ & \\
\hline Erythroxylaceae & Erythroxylum anguifugum Mart. & & $\mathrm{x}$ & $\mathrm{x}$ & $\mathrm{x}$ & $\mathrm{x}$ & & & \\
\hline \multirow[t]{2}{*}{ Euphorbiaceae } & $\begin{array}{l}\text { Alchornea castaneifolia (Humb. \& Bonpl. } \\
\text { ex Willd.) A. Juss. }\end{array}$ & $\mathrm{x}$ & & & & & & & \\
\hline & Alchornea discolor Poepp. & & $\mathrm{x}$ & $\mathrm{x}$ & $\mathrm{x}$ & $\mathrm{x}$ & $\mathrm{x}$ & $\mathrm{x}$ & \\
\hline \multirow[t]{13}{*}{ Fabaceae } & Albizia niopoides (Spruce) Burk. & $\mathrm{x}$ & $\mathrm{x}$ & & & & & & \\
\hline & Anadenanthera colubrina (Vell.) Brenan & $\mathrm{x}$ & $\mathrm{x}$ & & & & & & \\
\hline & Bauhinia rufa (Bong.) Steud. & & $\mathrm{x}$ & & & & & & \\
\hline & Bauhinia sp. & & $\mathrm{x}$ & $\mathrm{x}$ & & & & & \\
\hline & Bowdichia sp. & & & & & & & & $\mathrm{x}$ \\
\hline & Bowdichia virgilioides Kunth & & & & & & & & $\mathrm{x}$ \\
\hline & Dipteryx alata Vogel & $\mathrm{x}$ & & & & & & $\mathrm{x}$ & \\
\hline & Inga vera Willd. & & & & $\mathrm{x}$ & $\mathrm{x}$ & & & \\
\hline & Machaerium acutifolium Vogel & & & & & & & $\mathrm{x}$ & \\
\hline & Machaerium hirtum (Vell.) Stellfeld & & & & & & & & $\mathrm{x}$ \\
\hline & Mimosa adenocarpa Benth.. & $\mathrm{x}$ & & & & & & & \\
\hline & Platypodium elegans Vogel & & & & & & & $\mathrm{x}$ & \\
\hline & Vatairea macrocarpa (Benth.) Ducke & & & & & & & & $\mathrm{x}$ \\
\hline Lauraceae & Ocotea velloziana (Meisn.) Mez & $\mathrm{x}$ & & & & $\mathrm{x}$ & & & \\
\hline Lecythidaceae & Cariniana estrellensis (Raddi) Kuntze & & $\mathrm{x}$ & $\mathrm{x}$ & & $\mathrm{x}$ & & & \\
\hline \multirow[t]{4}{*}{ Malvaceae } & Chorisia speciosa A. St.-Hil. & $\mathrm{x}$ & & & & & & & \\
\hline & Guazuma tomentosa Kunth & $\mathrm{x}$ & $\mathrm{x}$ & & & & & & \\
\hline & Luehea paniculata Mart. & & $\mathrm{x}$ & & & & & $\mathrm{x}$ & \\
\hline & $\begin{array}{l}\text { (Mart. \& Zucc.) A. Robyns } \\
\text { Pseudobombax sp. }\end{array}$ & & $\mathrm{x}$ & & & & & $\mathrm{x}$ & \\
\hline Melastomaceae & Mouriri guianensis Aubl. & & & $\mathrm{x}$ & $\mathrm{x}$ & $\mathrm{x}$ & & & \\
\hline \multirow[t]{2}{*}{ Meliaceae } & Cedrella fissilis Vell. & $\mathrm{x}$ & & & & & & & \\
\hline & Trichilia elegans A. Juss. & & & & & $\mathrm{x}$ & & & \\
\hline
\end{tabular}

Revista Árvore, Viçosa-MG, v.35, n.5, p.1077-1087, 2011 
Tabela 1 - Cont.

Table 1 - Cont.

\begin{tabular}{|c|c|c|c|c|c|c|c|c|c|}
\hline \multirow[t]{2}{*}{ Moraceae } & Ficus gomeleira Kth et Bouché & & & $\mathrm{x}$ & $\mathrm{x}$ & $\mathrm{x}$ & & $\mathrm{x}$ & \\
\hline & Ficus pertusa L.f. & & & & & $\mathrm{x}$ & $\mathrm{x}$ & & \\
\hline Myrtaceae & Myrcia fallax (Rich.) DC. & & $\mathrm{x}$ & & $\mathrm{x}$ & $\mathrm{x}$ & & $\mathrm{x}$ & \\
\hline Phytolacaceae & Gallesia integrifolia (Spreng.) Harms & & & & $\mathrm{x}$ & $\mathrm{x}$ & & & \\
\hline Polygonaceae & Triplaris americana $\mathrm{L}$. & $\mathrm{x}$ & & & & $\mathrm{x}$ & & & \\
\hline Rhamnaceae & Rhamnidium elaeocarpum Reissek & $\mathrm{x}$ & $\mathrm{x}$ & & & & & $\mathrm{x}$ & \\
\hline \multirow[t]{6}{*}{ Rubiaceae } & Alibertia edulis (Rich.) A. Rich. ex DC. & $\mathrm{x}$ & $\mathrm{x}$ & & $\mathrm{x}$ & $\mathrm{x}$ & & $\mathrm{x}$ & \\
\hline & Alibertia sp. 1 & & & & $\mathrm{x}$ & $\mathrm{x}$ & & & \\
\hline & Alibertia sp. 1 & & & & & & & $\mathrm{x}$ & \\
\hline & Alibertia verrucosa S. Moore & & $\mathrm{x}$ & & & & & & \\
\hline & Rudgea viburnoides (Cham.) Benth. & & & & & & & $\mathrm{x}$ & \\
\hline & Tocoyena formosa (Cham. \& Schltdl.) K. Schum. & & & & & & & $\mathrm{x}$ & \\
\hline Salicaceae & Casearia gossypiosperma Briq. & $\mathrm{x}$ & & & & & & & \\
\hline Sapindaceae & Cupania casteinifolia Mart. & & & & & $\mathrm{x}$ & & & \\
\hline \multirow[t]{3}{*}{ Sapindaceae } & Dilodendron bipinnatum Radlk. & $\mathrm{x}$ & & & & & & $\mathrm{x}$ & \\
\hline & Magonia pubescens A. St.-Hil. & & & & & & & $\mathrm{x}$ & $\mathrm{x}$ \\
\hline & Talisia esculenta (A. St.-Hil.) Radlk. & $\mathrm{x}$ & $\mathrm{x}$ & & & & & & \\
\hline Sapotaceae & Pouteria glomerata (Miq.) Radlk & & $\mathrm{x}$ & & & & & & \\
\hline Simaroubaceae & Simarouba versicolor A. St.-Hil. & & & & & & & $\mathrm{x}$ & $\mathrm{x}$ \\
\hline Urticaceae & Cecropia pachystachya Trécul & $\mathrm{x}$ & & & $\mathrm{x}$ & & & & \\
\hline \multirow[t]{3}{*}{ Vochysiaceae } & Callisthene fasciculata Mart. & $\mathrm{x}$ & & & & & $\mathrm{x}$ & & $\mathrm{x}$ \\
\hline & Qualea multiflora Mart. & & $\mathrm{x}$ & & & & & & \\
\hline & Vochysia divergens Pohl. & & & $\mathrm{x}$ & $\mathrm{x}$ & $\mathrm{x}$ & & & \\
\hline
\end{tabular}

Protium heptaphyllum, Vochysia divergens, Calophyllum brasiliense, Alchornea discolor e Cariniana estrellensis (Tabela 1), que são descritas como espécies típicas de locais com ocorrência de inundações de longa duração (TORRES et al., 1994; TONIATO et al., 1998; IVANAUSKAS et al., 1997; OLIVEIRA-FILHO; RATTER, 2000; RODRIGUES; NAVE, 2001; LOBO; JOLY, 2000; MARTINS, 2001; MARQUES et al., 2003).

Os altos valores de importância dos indivíduos encontrados mortos nas parcelas 3, 4 e 5 podem ser explicados pela limitação causada pela inundação. Em áreas úmidas, a inundação inicia uma cadeia de reações conduzindo a redução das condições do solo, gerando anoxia, e isso dificulta ou impede a respiração radicular. Essas reações causam vários processos físicos, químicos e biológicos que possuem importância significativa para a vegetação. Plantas de áreas úmidas possuem várias características que permitem sua sobrevivência em solos periodicamente encharcados e acompanhados de mudanças químicas (PEZESHKI, 2001). Rodrigues e Shepherd (2001) consideraram a inundação ocorrente na faixa ciliar como instrumento natural de perturbação da vegetação. Essa perturbação seria consequência do extravasamento do rio, provocando o soterramento ou remoção periódica da serrapilheira, do banco de sementes e, mesmo, dos indivíduos jovens na faixa imediatamente ciliar ou do encharcamento prolongado do solo nos trechos de embaciamento, provocando a seletividade de espécies, com aumento da mortalidade de indivíduos nesses trechos.

Já nas parcelas 1, 2, 9 e 10 (Tabela 1) as espécies com maior valor de importância encontradas são tipicamente de regiões não inundáveis, entretanto nas parcelas 1 e 2 ocorrem a presença de espécies de áreas úmidas, como Cecropia pachystachya e C. estrellensis. Essas duas parcelas apresentam alta frequência e baixa duração de alagamento, contudo parte da parcela 2 está suscetível à maior duração de alagamento em inundações de grande magnitude, o que explica a presença de espécies de ambas as condições de alagamento. Nas parcelas com baixa frequência e duração de inundação ocorrem espécies tipicamente encontradas no cerrado em áreas não alagáveis, como Combretum leprosum, Talisia esculenta, Anadenanthera colubrina, Myracrodruon urundeuva, Triplaris americana, Machaerium hirtum, Callisthene fasciculata, Magonia pubescens e Curatella americana (SAPORETTI JR. et al., 2003; BALDUINO et al., 2006; NERI et al., 2007).

Revista Árvore, Viçosa-MG, v.35, n.5, p.1077-1087, 2011 
A espécie $C$. fasciculata apresentou alto valor de importância nas parcelas 2, 9 e 10. Essa espécie é uma árvore comum do cerrado em solos mesotróficos. No pantanal, essa espécie é encontrada no cerradão em depressões não inundáveis, considerando-se que a árvore tolera pequenas inundações sazonais e pode formar agrupamentos em torno de áreas alagáveis (HAASE et al., 1995).

\section{CONCLUSÃO}

As diferentes cotas topográficas em que as espécies analisadas se encontravam refletem o tempo de inundação a que suportam. Dessa forma, alterações no regime hidrológico causadas por barragens, principalmente aquelas relacionadas à magnitude das cheias, podem causar mudanças significativas nessas áreas e influenciar os processos de sucessão onde espécies de regiões secas invadirão áreas antes inundadas, alterando significativamente a composição natural da vegetação.

\section{REFERÊNCIAS}

ALVARENGA, S. M. et al. Estudo geomorfológico aplicado à Bacia do Alto Paraguai e Pantanais Matogrossenses. Boletim Técnico. Projeto RADAMBRASIL. Salvador: 1984. p.89-183. (Serie Geomorfologia, 1).

ANGIOSPERM PHYLOGENY GROUP - APG II. An update of the Angiosperm Phylogeny Group classification for the orders and families of flowering plants: APG II. Botanical Journal of the Linnean Society, v.141, p.399-436, 2003.

BALDUINO, A. P. C. et al. Fitossociologia e análise comparativa da composição florística do cerrado da flora de Paraopeba-MG. Revista Árvore, v.30, n.5, p.829-836, 2006.

BARBOUR, M. G.; BURK, J. H.; PITTS, W. D. Terrestrial plant ecology. 2.ed. Menlo Park: CA. Benjamin Cummings, 1987.634p.

BEDINGER, M. S. Forests and flooding with special reference to the White River and Ouachita River basins, Arkansas. Lakewood: USA. U.S. Geologic Survey, 1979. (Open File Report 79-680.
BRASIL. Ministério das Minas e Energia Projeto Radambrasil. Levantamento de Recursos Naturais. Rio de Janeiro; Folha SD 21, Cuiabá. v.26, 1982.

BREN, L. J.; GIBBS, N. L. Relationships between flood frequency, vegetation, and topography in a river red gum forest. Australian Forestry Research, v.16, p.357-70, 1986.

BROWER, J. E.; ZAR, J. H. Field and

laboratory methods for general ecology. Dubuque: W. M. C. Brown, 1984. 226p.

CAMPBELL, D. G.; STONE, J. L.; ROSAS JR., A. A comparison of the phytosociology and dynamics of three floodplain (Várzea) forests of known ages, rio Juruá, wester brazilian Amazon. Botanical Journal of Linnean Society, v.108, p.213-237, 1992.

CHAPIN, D. M.; BESCHTA, R. L.; SHEN, H. W. Relationships between flood frequencies and riparian plant communities in the upper Klamath Basin, Oregon. Journal American Water Resources Association, v.38, p.603-617, 2002.

CHRISTOFOLETTI, A. Geomorfologia fluvial - o canal fluvial. São Paulo: Edgard Blücher, , 1981. v.1. 313p.

DYNESIUS, M.; NILSSON, C. Fragmentation and flow regulation of river systems in the northern third of the world. Science, v.266, p.753-762, 1994.

FERREIRA, L. V. Effects of the duration of flooding on species richness and floristic composition in three hectares in Jaú National Park in floodplain forest in Central Amazonia. Biodiversity and Conservation, v.6, p.1353-1363, 1997.

FERREIRA, L. V. Effect of flooding duration on species richness, floristic composition and forest structure in river margin habitats in Amazonian blackwater floodplain forests: implications for future Design of protected areas. Biodiversity and Conservation, v.9, n.1, p.1-14, 2000.

FERREIRA, L. V.; ALMEIDA, S. S. Relação entre a altura de inundação, riqueza específica de plantas e o tamanho de clareiras naturais em uma floresta inundável de igapó, na Amazônia Central. Revista Árvore, v.29, n.3, p.445-453, 2005.

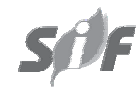

Revista Árvore, Viçosa-MG, v.35, n.5, p.1077-1087, 2011 
GAUCH, H. G. Multivariate analysis in community ecology. New York: Cambridge University Press, 1982.

GIRARD, P.; DA SILVA, C. L.; ABDO, M. Rivergroudwater interactions in the Brazilian Pantanal: the case of the Cuianá River. Journal of Hydrology, v.283, n.1, p.57-66, 2003.

GREGORY, S. V. et al. An ecosystem perspective of riparian zones. Bioscience, v.41, n.8, p.540-551, 1991.

HAASE, R.; HAASE, P. Above-ground biomass estimates for invasive trees and shrubs in the Pantanal of Mato Grosso, Brasil. Forest Ecology and Management, v.73, n.1, p.29-35, 1995.

HUGHES, F. M. R. The influence of flooding regimes on forest distribution and composition in the Tana River floodplain, Kenya. Jounal of Applied Ecology, v.27, p.475-491, 1990.

HUPP, C. R.; OSTERKAMP, W. R. Riparian vegetation and fluvial geomorphic processes. Geomorphology, v.14, p.277-295, 1996.

IVANAUSKAS, N. M.; RODRIGUES, R.R.; NAVE, A. G. Aspectos ecológicos de um trecho de Florestas de Brejo em Itatinga, SP: florística, fitossociologia e seletividade de espécies. Revista Brasileira de Botânica, v.20, n.1, p.139-153, 1997.

JUNK, W. J. The flood pulse concept of large rivers: learning from the tropics. Large Rivers, v.11, n.3; Arch. Hydrobiol. Suppl, 115/3, 261-280, 1999.

LIEBERMAN, M. et al. Small-scale altitudinal variation in lowland wet tropical forest vegetation. Journal of Ecology, v.73, n.2, p.505-516, 1985.

LOBO, P. C.; JOLY, C. A. Aspectos ecofisiológicos da vegetação de mata ciliar do sudeste do Brasil. In: RODRIGUES, R. R.; LEITÃO FILHO, H. F. (Eds.). Matas ciliares: conservação e recuperação. São Paulo: Editora da Universidade de São Paulo, 2000. p.143-157.
MARQUES, M. C. M.; SILVA, S. M.; SALINO, A. Florística e estrutura do componente arbustivoarbóreo de uma floresta higrófila da bacia do rio Jacaré-Pepira, SP, Brasil. Acta Botânica Brasilica, v.17, n.4, p.495-506, 2003.

MARTINS, S. V. Recuperação de Matas Ciliares. In: MARTINS, S. V.; VIEIRA, E. A. (Ed.).Viçosa, MG: Aprenda Fácil, 2001.143p.

MITSCH, W. J.; GOSSELINK, J. G. Wetlands. 7.ed. New York: Van Nostrand Reinhold Company, 1986. 722p.

MUELLER-DOMBOIS, D.; ELEMBERG, H. Aims and methods of vegetation ecology. New York: Willey \& Sons, 1974. 574p.

NERI, A. V. et al. Análise da estrutura de uma comunidade lenhosa em área de Cerrado sensu stricto no município de Senador Modestino Gonçalves, norte de Minas Gerais, Brasil.

Revista Árvore, v.31, n.1, p.123-134, 2007.

NILSSON, C.; SVEDMARK, M. Basic principles and ecological consequences of changing water regimes: riparian plant communities.

Environmental Management, v.30, n. 4, p.468-480, 2002.

OLIVEIRA-FILHO, A. T.; RATTER, J. A. A study of the origin of central Brazilian forests by the analysis of plant species distribution patterns. Edinburgh Journal of Botany, v.52, n.2, p.141-194. 1995.

PCBAP - Plano de Conservação da Bacia do Alto Paraguai, v.2. 1997.

PEZESHKI, S. R. Wetland plant responses to soil flooding. Environmental and

Experimental Botany, v.46, p.299-312, 2001.

POFF, L. N. et al. The natural flow regime: a paradigm for river conservation and restoration. BioScience, v.47, p.769-784, 1997.

POLZIN, M. L.; ROOD, S. B. Effects of damming and flow stabilization on riparian processes and black cottonwoods along the Kootenay River. Rivers, v.7, p.221-232, 2000. 
RODRIGUES, R. R.; NAVE, A. Heterogeneidade florística das matas ciliares. In: RODRIGUES, R. R.; LEITÃO-FILHO, H. F. (Ed.). Matas

ciliares: conservação e recuperação. 2.ed. São Paulo: EDUSP/FAPESP, 2001. p.45-71.

RODRIGUES, R. R.; SHEPHERD, G. J. Fatores condicionantes da vegetação ciliar. In: RODRIGUES, R. R.; LEITÃO-FILHO, H. F. (Ed.).

Matas ciliares: conservação e recuperação. 2.ed. São Paulo: EDUSP/FAPESP, 2001. p.101-105.

SAPORETTI JR, A. W.; MEIRA NETO, J. A. A.; ALMADO, R. P. Fitossociologia de cerrado sensu stricto no município de Abaeté-MG. Revista Árvore, v.27, n.3, p.413-419, 2003.

SEVERI, W. A pesca do Rio Cuiabá: características e perspectivas. In: FERREIRA, M. S. F. D. (Ed). O Rio Cuiabá como subsídio para a educação ambiental. Cuiabá: EDUFMT, 1999. p.63-102.
TEVERSHAM, J. M.; SLAYMAKER, J. Vegetation composition in realtion to flood frequency in Lillooet River Valley, British Columbia. Catena, v.3, p.191-201, 1976.

TONIATO, M. T. Z.; LEITÃO-FILHO, H. F.; RODRIGUES, R. R. Fitossociologia de um remanescente de Floresta higrófila (Mata de brejo) em Campinas, SP. Revista Brasileira de Botânica, v.2, n.21, p.197-210, 1998.

TORRES, R. B.; MATHES, L. A. F.; RODRIGUES, R. R. Florística e estrutura do componente arbóreo de mata de brejo em Campinas, SP. Revista Brasileira de Botânica, v.17. n.2, p.189-194, 1994.

TUCCI, C. E. M. (Ed.). Hidrologia - ciência e aplicação. 2.ed. Porto Alegre: Universidade Federal do Rio Grande do Sul, 2001. 
